\title{
What We Learned about Mentoring Research Assistants Employed in a Complex, Mixed-Methods Health Study
}

Lori E. Weeks

Dalhousie University

Michelle A. Villeneuve

University of Sydney

Susan Hutchinson

Dalhousie University

Kerstin Roger

University of Manitoba

Joan Versnel, \& Tanya Packer

Dalhousie University

\begin{abstract}
We investigated the experiences of research assistants in their dual role as both employees and trainees, when they were employed in a complex, mixedmethods, Canadian study on the everyday experience of living with and managing a chronic condition. A total of 13 research assistants participated in one or more components of this study: a survey $(n=11)$, focus group interview ( $n$ $=7$ ), and/or individual interview $(n=13)$. Thematic analysis identified two key themes: what faculty mentors should provide to research assistants before they begin their work, and what faculty mentors need to know in order to effectively offer ongoing support to research assistants. Our results provide valuable insights for new and experienced faculty members who employ research assistants and for research assistants employed in funded research projects. Our results can inform the development of regulations to ensure that research assistants have greater protection as both trainees and employees.
\end{abstract}




\section{Résumé}

Lors d'une recherche canadienne complexe à méthodologie mixte portant sur le quotidien des gens qui vivent avec une condition chronique ou qui doivent en gérer les aspects, nous avons exploré le double rôle des adjoints à la recherche, à la fois comme employés et comme stagiaires. Au total, 13 adjoints à la recherche ont participé à au moins une composante de cette étude : un sondage $(n=11)$, une entrevue de groupe $(n=7)$, une entrevue individuelle $(n=13)$. Une analyse thématique a pu repérer deux thèmes principaux : ce que les mentors du corps professoral doivent savoir afin d'aider efficacement et de façon soutenue les adjoints à la recherche, et ce qu'ils doivent leur fournir avant le début des travaux. Nos résultats fournissent de précieux éclaircissements pour de nouveaux membres et des membres chevronnés du corps professoral qui emploient des adjoints à la recherche, ainsi que pour des adjoints à la recherche qui travaillent à des projets subventionnés. Nos résultats informent les responsables de l'élaboration des règlements afin de s'assurer que les adjoints à la recherche disposent d'une protection élargie en tant que stagiaires et employés.

Students are mentored in the research process by faculty members in many contexts within higher education. This can involve: faculty members mentoring graduate students throughout the process of completing a thesis or dissertation (Earley, 2007; Humble, Solomon, Allen, Blaisure, \& Johnson, 2006); graduate students participating, in an unpaid capacity, in research collaborations with faculty members on research that results in peer-reviewed publications (Paglis, Green, \& Bauer, 2006); students working on a faculty member's research as a class project (Wulf-Andersen, Holger Mogensen, \& Hjort-Madsen, 2013); or an independent study on a topic of interest to the student (Moore, Scarduzio, Plump, \& Geist-Martin, 2013). In this study, we focus on a mentoring context that has received less research attention: situations in which faculty members employ research assistants (RAs) to work on faculty-directed, funded research (Rossouw \& Niemczyk, 2013). While these RAs are employees, their positions are also developmental and educational, which adds complexity to a traditional employee-employer relationship (Edwards, 2009; Rossouw \& Niemczyk, 2013). It is exploring this dual role of employee and trainee that guides our work and contributes to our understanding of how to provide a positive educational and employment experience to RAs.

While knowledge generated from research is a key measure of a faculty member's performance (Hobson, Jones, \& Deane, 2005), large-scale research endeavours typically rely on RAs, who support researchers in the implementation of the study objectives. Indeed, research funding allows for the hiring of RAs to support time-poor faculty members in the implementation of time-intensive research tasks (Hey, 2001). Large-scale health research projects can involve the implementation of complex study protocols and interaction with study participants, which invariably require the involvement of RAs. Research assistants engage in diverse tasks requiring advanced skills, including the ability to recruit and retain study participants (Cambron \& Evans, 2003), and tasks requiring specific technical and analytical expertise (Hobson, et al., 2005). Consequently, investigators running large-scale studies take on the role of mentor to support and manage an array of activities engaged in by paid RAs. 
Until quite recently, little peer-reviewed research focused on RAs (Edwards, 2009; McGinn, Niemczyk, \& Saudelli, 2013). While research results are shared through various mechanisms, knowledge gained through the implementation of research projects is rarely shared (Cambron \& Evans, 2003; Earley, 2007). A recent special issue in the Journal of Research Practice focused on RAs (McGinn \& Niemczyk, 2013), although not all of the articles examined paid RAs. In this issue, Naufel and Beike (2013) focused on the ethical treatment of RAs and identified several elements to include in a RA Bill of Rights as a preliminary step in ensuring that RAs are treated ethically. Our research can contribute to this type of document.

While students participate in research assistantships for many reasons, a primary one is to learn new research skills (Niemczyk, 2010). There is some emphasis in the literature on the importance of training for RAs to implement research involving human participants. Training can help faculty mentors and individual RAs to identify research activities that fall outside their specific abilities, interests, and preferences (Naufel \& Beike, 2013). Cambron and Evans (2003) examined RA experiences when implementing large-scale clinical trials and offered recommendations to improve future training methods. They emphasized the need to train RAs to better manage the boundaries of their role and their responsibilities with respect to implementing data-gathering protocols with participants.

Other researchers have identified strategies that faculty members who hire and manage research staff can use to ensure high-quality research implementation efforts (Kang, Davis, Habermann, Rice, \& Broome, 2005). Hobson and colleagues (2005), motivated by their own role as research managers, described the role and contribution of RAs as knowledge workers and highlighted the need for research that recognizes the ways in which RAs contribute to knowledge generation. Similarly, other authors have argued that RAs should be viewed as critical members of a research team, and they have recommended methods of research management that acknowledge the shared responsibility of investigators and RAs rather than approaches that perpetuate power imbalances (Miller \& Stephens, 1998; Nishimura et al., 2003).

While faculty members are well trained in planning and implementing research projects, graduate school gives them less preparation for mentoring RAs (Madden, 2009). In this study, we investigate the experiences of RAs employed in a complex, mixed-methods, Canadian study in which we examined the everyday experience of living with and managing a chronic condition. We focus specifically on what we learned from RAs about how to effectively mentor RAs in their dual role as trainees and employees. In the next section, we describe the context in which our RAs worked, including their duties, the training they received, and the ongoing support that was provided for them to implement study protocols. More detail about the methods used in this study can be found in a published protocol paper (Versnel et al., 2013).

\section{RA Duties, Training, and Ongoing Support Provided}

We hired a total of 15 RAs across three study sites in three different Canadian provinces. We invited applications from potential RAs with a minimum of a bachelor degree that included the completion of a research project. Additional criteria for hiring RAs included prior research experience, interpersonal skills, strong communication skills, organizational skills, and the ability to manage one's time. Although we only hired female RAs, no gender criteria existed for employment as a RA. 
The RAs worked on three nested studies: a cross-sectional survey, a longitudinal cohort study, and case studies. The RAs participated in a wide variety of research activities, including: assisting with literature reviews; creating data collection instruments; contributing to Research Ethics Board submissions; collecting various types of data; participating in data management, entry, cleaning, coding, and analysis; contributing to reports and presentations; and organizing research meetings. Under the supervision of faculty investigators, tasks of two RAs included training other RAs, peer mentoring, and co-ordinating other RAs across the study sites. Each RA had varying degrees of involvement within the study, depending on the timing of their involvement in the study, as well as their interests, skills, knowledge, and prior professional experience. Thus, they each had a unique set of roles and responsibilities.

\section{Cross-sectional Survey}

The cross-sectional survey involved self-reporting, and most participants completed it online. We also made a paper-and-pencil version and a telephone interview available. Research assistants from one study site administered the survey via telephone with a small number of respondents who had selected that format. The survey took approximately 1.5 hours to complete, and a total of 787 people completed it. All RAs received training on the audio and online technologies used to collect and record data. The RAs also received training on procedures to maintain respondent confidentiality and anonymity.

\section{Cohort Study}

A prospective, longitudinal cohort study design added depth to knowledge gained from the cross-sectional survey. We drew a sequential sample from those who, on completion of the survey, expressed interest in participating in future research. The sample included 118 adults aged 17 to 65 and 18 parents of children aged five to 16. RAs conducted monthly telephone interviews with study participants for a total of 11 months. Each interview took approximately 45 minutes. Data collected took the form of semi-structured and openended questions, augmented with standardized surveys. During the monthly telephone interviews, RAs simultaneously conducted interviews and entered data into an online form. The RAs used a tracking sheet to record the status of interviews and to schedule future interviews.

The co-principal investigators developed a cohort study training manual with support from a RA with expanded duties who served as a research co-ordinator. The research co-ordinator held ongoing responsibility for updating the training manual and providing technical support to RAs throughout the implementation of the cohort study, with supervision provided by study investigators at each of the three data collection sites. The RA training manual described all duties and procedures related to data collection. All RAs received a copy of the training manual one month prior to the initial four-hour group RA training session, which took place approximately one month prior to the beginning of data collection. RAs attended either in person or via videoconference. Investigators responsible for supervising RAs at each site also attended the session during which the principal investigators and research co-ordinator provided an overview of the cohort study, answered questions concerning implementation of the study protocol, and provided RAs with hands-on practice using data entry tools, participant tracking tools, and 
protocols for confidential file transfers. Three RAs, hired after the group training session, received training in a separate session by the research co-ordinator.

The research co-ordinator worked in concert with the study investigators at each of the three sites to troubleshoot during early implementation and to provide ongoing support throughout the full implementation of the cohort study. Support included email, Skype, or telephone conversations, and generally occurred on an individual basis. The research coordinator made updates to the training manual based on feedback received from the RAs during early implementation of the study, and RAs received updated versions of the training manual. For example, RAs raised questions about technical aspects of data entry, data management, encryption software, and completing transfers of confidential large files. Research assistants requested clearer procedures for obtaining informed consent from participants who appeared to have deteriorating cognition. Other feedback included strategies for backing up data in the event of interruptions to Internet access. A total of eight versions of the RA training manual reflected the collective insights gained by the RAs.

Six months after the initial group training, the RAs attended a face-to-face meeting to share experiences with each other and the study investigators. During this meeting, many RAs expressed being deeply affected by the openness of study participants in sharing personal information about their everyday experience of managing a chronic condition. Some RAs found it challenging to accept their role as data gatherer while developing rapport through regular contact with study participants. Others expressed distress at being "observers and data collectors" rather than providers of "assistance." At this meeting, investigators recognized the need to provide additional guidance and opportunity for RAs to develop strategies for managing the boundaries of their role with study participants. Investigators created an additional training session that involved all RAs and enabled participation via teleconference for RAs working across the data collection sites.

\section{Case Studies}

In the qualitative case studies, RAs captured and documented the complexity of living with and managing a chronic condition from the perspective of adult participants, the parents of child participants, people providing unpaid support to study participants, and health-care providers. RAs gathered interview data using semi-structured interview guides. RAs with prior experience in qualitative interviewing conducted these interviews face-to-face in participants' homes or places of work, or via telephone. Several RAs transcribed the interview data verbatim, including clarifying questions about the recording with the RA who had conducted the interview. The sample included 15 adults aged 17 to 65 and six parents of children aged five to 16.

Training for all RAs involved in case study data collection took place at a three-hour face-to-face meeting. A post-doctoral fellow provided training, including an overview of the case studies and instruction in qualitative interviewing techniques. Research assistants had the opportunity to review the interview guides and have their questions answered. The study investigators at each data collection site, the post-doctoral fellow, and a research co-ordinator provided ongoing supervision for the RAs involved in the case studies, either in person, via telephone, or by email. The research co-ordinator again provided technical support, and the post-doctoral fellow reviewed interview transcripts and provided individual feedback and coaching to the RAs on their interview skills. Following preliminary data 
analysis of the interviews, the post-doctoral fellow produced individual interview guides for follow-up interviews with study participants, again individually coaching each RA.

\section{Methods}

The investigators of the RA study are a subset of the investigators involved in the larger research project examining the everyday experience of living with and managing a chronic condition. No RAs participated in collecting or analyzing data from other RAs in this study. We obtained approval from the Research Ethics Boards at the institutions of each investigator involved in the RA study. While most of the investigators in the RA study had direct RA supervisory responsibility in the larger project, one investigator who had no prior involvement in hiring, supervising, or mentoring RAs in the larger study facilitated all RA recruitment and data-gathering activities for this study. This investigator also took responsibility for member-checking and de-identifying data prior to sharing it with other investigators.

\section{Participant Recruitment}

All RAs, including one RA with primarily data analysis duties, received an emailed invitation to participate in the RA study; this invitation included an information letter and a consent form. We ensured that the RAs understood that their participation in the RA study was entirely voluntary and that it would occur outside their role, responsibilities, and paid time with the research project. The investigator responsible for RA recruitment and data collection responded to all questions raised. The RAs received no compensation for participation in the RA study. Out of a total of 15 RAs, two declined to participate in the RA study, resulting in a sample of 13 .

\section{Data Collection}

The 13 RAs participated in one or more of the three components of the RA study: a survey $(n=11)$, a focus group interview $(n=7)$, and an individual interview $(n=13)$.

Survey. The RAs received and returned a short survey by email. Data collected included background information on age, education, RA roles within this project, previous work and volunteer experience, previous experience working with individuals with chronic conditions or disability, and prior research experience.

Focus group interviews. Following focus group procedures as specified by Kruger and Casey (2000), we held two different focus group meetings to accommodate the RAs' work and/or education schedules. In one focus group, two RAs participated by telephone, and in the other focus group, one RA participated by telephone and four RAs participated face-to-face. Topics that guided the conversation during the focus groups included: challenging aspects of being a RA and response to these challenges; what RAs learned; and recommendations for future training, mentorship, and supervision of RAs. All RAs had an opportunity to respond to each question. Each focus group lasted approximately 70 minutes. Verbatim transcripts were prepared from the audio-recorded conversations.

Individual interviews. All 13 RAs completed an individual semi-structured interview either by telephone or in person. After obtaining permission from each RA, the investigator conducting the interviews audio-recorded each interview. The investigator first 
provided a summary of the key results from the focus group meetings and then invited each RA to provide feedback, such as surprising results, items with which the RA disagreed, or issues of importance to the RA. The investigator then asked a series of questions about: (i) how the RA experienced the implementation of the study (e.g., preparation and training as a RA, supervision received, responsibilities, and challenges or dilemmas encountered), (ii) strategies used in navigating and managing the challenges and multiple relationships associated with being a RA (e.g., emotions and coping with their emotions; strategies used in managing relationships with the investigators, other RAs, and research participants); and (iii) any transformation experienced through conducting research (e.g., being part of a research team, the everyday lived experience of having a chronic condition).

\section{Data Management and Analysis}

After signing a confidentiality agreement, a professional transcriptionist prepared a verbatim transcript from each audio-recorded focus group and individual interview. The investigator who conducted the RA data collection removed from the transcribed data all identifying information (e.g., names, employment site, supervisor names). The RAs had the opportunity to review transcripts and remove or clarify any text. The investigator entered the de-identified data into NVivo, a qualitative software program used to support the organization and coding of data. Only after the completion of this process did other investigators have access to the data.

To begin the data analysis process, we first identified any text focused on issues related to training and providing ongoing support to RAs. Then, we used a thematic analysis approach, which is a form of pattern recognition allowing for themes to emerge directly using inductive coding (Fereday \& Muir-Cochrane, 2006). Thematic analysis is particularly useful in understanding influences and motivations related to how people respond to events (Luborsky, 1994). In the case of this study, our interest was in obtaining feedback from RAs about their decision to become a RA and their experiences of being a RA in a study focused on people living with chronic health issues. Thus, thematic analysis lent itself well to exploring the experiences of RAs employed to work on a large and complex health research project. The coding process involved generating initial codes, searching for themes, reviewing themes, and defining and naming themes that resulted in thematic codes that represented patterned responses within the data set (Braun \& Clarke, 2006). One investigator followed this process to identify the main themes and subthemes that emerged from the transcripts. After finalizing the themes and subthemes, one investigator coded all the transcripts in NVivo. In some instances, text appears in more than one theme or subtheme, due to multiple themes emerging within the same passage of text.

\section{Results}

\section{RA Characteristics}

In order to ensure the confidentiality and anonymity of the RAs, we only provided aggregate information about their characteristics. Although the RAs had a mean age of 36, we identified two distinct groups of RAs based on their age, training, and professional experience. One group consisted of RAs under age 35, and almost all of these RAs were concurrently enrolled in a graduate degree $(n=8)$. While many of these younger RAs had 
experience working on other research projects and/or had relevant volunteer experience, they tended to have less professional experience than the other group consisting of RAs aged 35 or older $(n=5)$. All the RAs in this older group had completed a degree and/or professional credential at least 15 years prior to becoming a RA with this project, and most had more than one undergraduate and/or graduate degree. The older RAs came to the project with extensive experience working in various health and human-service settings.

\section{Themes and Subthemes}

In Table 1, we include a summary of the themes and subthemes that emerged from the data, along with the number of transcripts that reflected each subtheme.

Table 1.

Summary of Themes and Subthemes

Themes and Subthemes

Number of

transcripts

$n=15$

Theme 1: Training

What faculty mentors should provide to RAs before they begin their work.

1.1 Provide guidance to RAs about appropriate interactions with research participants who have health challenges.

1.2 Provide hands-on training for collecting data.

1.3 Ensure that the roles and expectations of all team members are clear.

1.4 Provide training for all forms of technology used in the study.

1.5 Ensure that RAs have a clear understanding of the importance of the study and plans for dissemination of the results.

Theme 2: Ongoing Support

What faculty mentors need to know to effectively provide ongoing support to RAs.

2.1 Implement a system of delivering frequent feedback to RAs.

2.2 Understand the motivators for becoming a RA and the rewards that RAs receive.

2.3 Provide opportunities for RAs to feel like an integral part of the research team.

2.4 Encourage opportunities for peer mentorship among RAs.

2.5 Seek input from RAs throughout the study.

2.6 Ensure that a smooth transition occurs between RAs.

2.7 Educational credentials are not the only important factor in determining who will be an effective RA.

2.8 Pay for all hours worked and expenses incurred. 4

Note: The number of transcripts is made up of 13 individual interviews and two focus group interviews. 
Theme 1, RA training: What faculty mentors should provide to RAs before they begin their work. Issues related to the RA training manual for the cohort portion of our study emerged as an important overall training matter for the RAs, especially as the manual became a living document, with new information added as needed. Thus, we did not define it as belonging to one of the subthemes below, but as a key factor that spanned various subthemes.

I thought it was very helpful. I think that was done very well. . . If there were ever any adjustments that needed to be made, I mean obviously you can't think of all the gaps at the beginning, right.... [T] hroughout the interview process, once that became an issue for some of them, they added a section, and I liked how it was set up. It was easy to follow, easy to understand. (RA6)

1.1 Provide guidance to RAs about appropriate interactions with research participants who have health challenges. This subtheme emerged in all individual interviews and both focus groups. RAs described various challenges and insights into the unique relationship between them and study participants. It appeared particularly challenging for some RAs to respond to participants who provided responses indicating a particularly poor health or quality of life, especially if they appeared upset, or if the RAs completed data collection face-to-face instead of over the telephone.

I think those lines of kind of the reciprocal relationship get blurred because while it is your job, people are revealing very, very intimate details about themselves with you, and so it doesn't feel like a professional relationship no matter how cordial and how formal you are, you know things that many people don't know about this person. So you do feel a certain type of closeness. (Focus Group 1)

Many RA discussed challenges in knowing how to interact with participants. While RAs recognized the importance of establishing rapport with participants, many experienced difficulties in doing this: "I feel like I was so concerned about being a researcher and scientific that maybe I didn't give enough of the human part of me" (Focus Group 2). Several RAs discussed finding a comfortable space between being detached and being a friend or helping professional, or being compassionate without stepping over a boundary. "For me it was always emphasizing that I am here to record your views and to do that as accurately as I can because they are important" (RA4). However, this balance could be difficult to achieve, especially given participants' expectations.

At times I thought what was prescribed as the role of the RA or the researcher was sometimes at odds with what the participant expected or wanted or needed. ... So we have this job where we are going to speak with people every month, and we are going to serve as the recorder of their voice and of their experience, and to me the biggest challenge was to convey to them that was my role. (Focus Group 2)

Several RAs benefited from direct guidance received from the investigators about the role of the RAs. The addition of a special training session on this topic gave RAs a great deal of assistance and reassurance in clarifying their role when navigating the data collection process with participants. 
They held a special training session for us, and they really took a lot of time to think about preparing for that, and make sure that they took the time to listen and respond to our concerns, which was really nice. And I really learned a lot from that experience, I would say, in terms of the difference between the role of a researcher and the role of, say, a friend or a medical professional or even a listener. (RA2)

The end of the data collection period also posed challenges for some RAs in interacting with participants. "I tell these participants we have done our job and we've done what we can do now, and now it's time to hand this over to the researchers and let them do their part" (RA4). Some RAs desired a way to continue their involvement with study participants dealing with chronic health challenges. "It was very sad that I wasn't going to be able to follow up with people and see how they were doing" (RA10).

1.2 Provide hands-on training for collecting data. Several RAs desired an opportunity to practice collecting data to become more familiar with the questionnaires and how to ask the questions, and especially how to ask open-ended questions appropriately, before they began collecting data from participants. "You really don't benefit that much from a training session until you experience doing an interview yourself" (RA3). RAs provided information on what such a session could entail. "Maybe even do, like, a simulated typical interview, what it's going to be like, and then give people a chance to process it, then ask questions" (RA3). Some RAs desired a long period of time to practice collecting data. "I almost wish that we could do month one and use that as our learning and then talk as a group and say, okay, here were the issues" (Focus Group 1). In addition to practice data collection sessions, RAs also identified the benefits of being able to observe another RA.

I had the opportunity to see several different people administer the interviews. . . That was very educational to see different strategies that people had for reacting to emotionally charged things, different ways that people had, you know, different paces that people set for their interviews, different ways of setting up the interview space. That was great, I really enjoyed that. (RA2)

In addition to practice data collection sessions, many RAs also wanted to have an ongoing process whereby they would receive feedback on the quality of the data collected.

That would have been really helpful if, let's say, the first couple of interviews, even, just go through the interview and then have them say, you know, in this situation, maybe don't say your personal information, or maybe here when the person is getting emotional, you can say ... You are actually going through it and it's going to stick better, I think. (RA6)

Other RAs felt that a quality assurance process should occur throughout the entire data collection process. "So maybe if there was, like, a set time-like, after so many interviews, you need to come and see this person and review these. ... I would want the feedback" (RA7). Both less-experienced and more-experienced RAs desired this type of feedback.

And I even think, coming into it, you know, a fairly seasoned interviewer, it still would have been helpful to me just to know that I'm doing this the way you would like me to do it or that perhaps there were tips or tricks to help me overcome maybe some stumbles I would have had. (RA10) 


\subsection{Ensure that the roles and expectations of all team members are clear.} RAs preferred an environment with clear roles and expectations and in which they understood to whom to report. "And so I think some of the trouble I ran into was just different expectations from different folks and not knowing who you were serving, you know. I think that would be my biggest challenge" (RA13). In some instances, RAs would have preferred more support than they received, but they understood the expectation to work as independently as possible. "She just has a way of supervising that is so affirming of our skills that she would trust our judgement on certain things. And, of course, I would use my judgement" (RA9). Some RAs understood the intent to create a collegial versus a hierarchical environment.

I thought the people were very much on the same page and that it was quite an open dialogue, and that people wanted to hear others' opinions and get input, but that people had quite clearly defined roles still, so there's obviously still a hierarchy but it was, there was a good exchange of ideas within that. (RA11)

In addition to clearly understanding their own role, the RAs also wanted to know the role of the other team members. "So, fully understanding what a research assistant does, what a research associate does, what a postdoc does, and what a project manager does, I think is key" (RA3). Challenges in achieving this emerged during this multi-year project, with some research staff turnover. One RA who joined the team much later than others indicated: "There just seemed to be an incredible number of people involved in the project, and for the most part I had no idea who 90\% of them were" (RA12).

1.4 Provide training for all forms of technology used in the study. RAs had to use various forms of technology to complete their duties. Some RAs appeared very comfortable with using these technologies, while others required additional training and support. RAs had divergent suggestions about the amount of training and support needed to solve technical issues.

For me, paper-based would have been so comforting and then to transcribe it ... so I had a huge learning curve with learning how to do drop files and, you know, all the little things that probably are so easy to [other RAs] ... the lack of technology support for me, sort of a novice, was the biggest stressor for me. (Focus Group 1)

As RAs desired to collect high-quality data, some appeared to be nervous using various technologies, especially early in the data collection process.

I was apprehensive on the first interview, but I've done enough of them myself that I wasn't nervous about interviewing people. I was simply nervous about interacting with the software and making sure I had everything in place to collect the data. But after the first couple it was very easy, very straightforward, so not much anxiety over that. (RA10)

Some RAs perceived that they experienced technological challenges due to their age or to having had little prior experience with technology. 
That didn't seem to be a problem for them, and I think maybe it's a generational thing. ... For me it would have been a little more focus on getting that stuff up and running ... so all these things took so much time, so if there was a little more focus on that for old RAs like me. (RA4)

RAs valued training about what to do if unusual circumstances arose during data collection, such as the Internet going down.

I really appreciated [a research co-ordinator] being there to ask me every step of the way what to do when this happens, what to do when you lose the Internet in the middle of an interview. What do you tell somebody? So I see logistics and technical training and troubleshooting as something that should be a part of training. (Focus Group 2)

1.5 Ensure that RAs have a clear understanding of the importance of the study and plans for dissemination of the results. The RAs identified the importance of being able to articulate the value of the study to participants.

I think a lot of them, or at least some of them [i.e., study participants], feel like this is a bit like a waste of their time, so it's hard to convince them of the merits of the study. ... It's definitely been hard to articulate that on my own when people ask, and to keep articulating it in different ways as the months go on. (Focus Group 1)

The RAs also wanted to ensure that the participants' voices would be heard in the study results, irrespective of the methods used to disseminate the results. They appeared most concerned that qualitative results be disseminated.

I was always a bit concerned that because of the amount of data that we were collecting, they would never be able to use all that data, so I was more concerned about people, like, giving all kinds of information and then never seeing it anywhere, and wondering, Well why did they ask me all of that if I don't see it, just because of the logistics of never being able to present so much, especially the qualitative data we collected. (RA2)

Theme 2, RA Support: What faculty mentors need to know to effectively provide ongoing support to RAs.

2.1 Implement a system of delivering frequent feedback to $R A s$. Issues surrounding feedback to RAs emerged as another important theme both in initial RA training and in providing ongoing support through effective feedback practices. We identified several approaches to requests for feedback initiated by a RA, as well as opportunities to provide feedback initiated by an investigator.

The RAs identified the desire to receive feedback from investigators. The RAs suggested various ways to accomplish this, such as face-to-face meetings, telephone conversations, or email. "I think it would have been helpful to sort of have a constant fit of somehow feeding back some of how that was going, not by email, but by speaking" (RA13). One RA suggested that a mechanism to anonymously solicit feedback from investigators would be helpful. 
And there are definitely things that I would never say to study investigators that I think very strongly, and it's nice to have this opportunity where we can report things that we experience or observe anonymously, but that's very rare. (RA2)

In addition to investigators ensuring that RAs have opportunities to request feedback, we identified that RAs also desired active feedback, which does not depend on the RA seeking out an investigator to have questions answered. "You need to actively support them instead of telling them, When you have a problem, please come find me" (Focus Group 1). This could involve individual meetings or check-ins with the RA to deal with any immediate issues as they arise, or meetings as a group of RAs with an investigator for things that could not wait until a scheduled meeting. "Just talking to her and asking her, like, feedback on the interviews that I did do, and she would, sometimes I didn't even need to ask, she would just give them to me, so that was good" (RA5).

Challenges emerged due to having RAs employed at three data collection sites. "We would talk a lot as we were working there throughout the day, and we would have our weekly team meetings, whereas other RAs at the other sites didn't have that" (RA11). In addition to working at various sites, some RAs completed data collection with participants outside of regular office hours to accommodate the participants' schedules. This could create challenges, especially the ability to provide immediate feedback to RAs during data collection sessions. "Because I was working from home in the evenings ... I didn't really feel like I was working with them per se, but certainly I still felt like they were a resource and a support if I needed them" (RA12).

\subsection{Understand the motivators for becoming $a \mathrm{RA}$ and the rewards that} $\boldsymbol{R A s}$ receive. RAs wanted investigators to have a thorough understanding that RAs are not only employees working for wages, but also trainees seeking to gain experience for a plethora of professional and personal reasons. Working with our study provided RAs with the opportunity to enrich their educational experience in general. "Being a research assistant with [study name] served to clarify for me things ... [it was] an opportunity to act on, to apply, to integrate my accumulated learnings" (RA9). In addition, the RAs wanted to increase their quantitative and/or qualitative research skills, improve their skills in writing a literature review, experience working with a large research project, and use the experience to help them decide on a career path. "I gained a much better understanding of how a large-scale research project works" (RA11).

In addition to specific goals related to research, some RAs hoped to work in the future with people with various health challenges. The RAs gained a great deal of knowledge about people living with chronic health conditions, people living with disabilities in general, and the support networks of the participants. Some RAs acquired greater insight into the realities of peoples' lives and how each had a unique experience. "I think I had also developed in my mind of what MS is, and I'm really glad that's being validated now" (RA2). They gained an enhanced understanding of challenges related to an inaccessible environment, challenges related to finances and employment, and various programs in the community. "I didn't even know that program was available, or I didn't know this existed" (RA7).

Our RAs also learned a great deal about themselves, in some cases gaining increased perspective on their own lives. "When you actually hear that voice first-hand, it's really 
powerful. It makes you very thankful for what you have" (RA4). Some RAs appeared to be quite surprised that they so much enjoyed interviewing participants. "You get to talk to these people, and they make you laugh, and they have these amazing stories that they're telling you.... They gave me strategies for my own day-to-day activities, you know" (RA6).

2.3 Provide opportunities for $R A$ s to feel like an integral part of the research team. It became evident that RAs appreciated any efforts to help them feel like part of the research team. The RAs wanted to know how their work fit together with the work of others.

Did I feel like I was part of a team? Well, yes and no, I mean I knew that. . . . I know that we're all working towards I think the same goals, but the work was very independent, so, you know, it wasn't really that sense of camaraderie, so to speak. (RA1)

However, another RA worked on multiple tasks that provided her with a broader understanding of the project.

I didn't design the study. I'm simply taking this information that was, you know, pre-set for me, and then collecting it, and then to see what happens with it. I love to see that interaction myself, which is why I'm actually quite glad I get to do both sides of it, because I do like to see how things are coming out. Because you can get a sense when you're collecting the data, when you actually correlate and look at the numbers, but then you have something to go on to say, yeah, that matches what I had or no, that looks very different to what I, you know, was hearing. That's interesting. (RA10)

A broad knowledge of the project could even contribute to the quality of the data the RAs collected.

It gives, you know, just feeling like you were part of something that had so much value and that everybody was so invested in, it just gave you the incentive or, you know, whatever you needed to really go with it and make sure you got the data. (RA8)

The RA training manual helped in this regard, as it included a lot of background about the goals and objectives of the study. Although meetings and other forms of communication were time-consuming, RAs wanted to be a part of them. "She was able to organize enough teleconferences or face-to-face meetings that we really definitely felt part of a team" (RA8). Face-to-face meetings in particular seemed to be especially valuable to the RAs.

I would have liked to have been involved not to speak but perhaps to listen in on some of the meetings that went on, just to get an idea of the big picture. Because sometimes I think I didn't always have an idea of the big picture, and that helps me understand. (RA13)

2.4 Encourage opportunities for peer mentorship among $R A$ s. Research assistants in both focus group interviews and in nine individual interviews discussed the value of the opportunity for peer mentorship among RAs. While some opportunities for 
peer mentorship existed, the RAs wanted to have an ongoing forum to obtain peer mentorship. "What I wish was that as RAs we could have a weekly teleconference or conference call and talk as a group" (Focus Group 1).

The RAs identified many things they could learn from other RAs, such as how to communicate with participants with various types of chronic health conditions and personalities, how to use various forms of technology, and who to contact about specific issues. As not all the RAs began employment at the same time, those newer to the project appreciated learning from more experienced RAs. In general, they valued the feeling of support from other RAs.

Just having someone who is going through something similar and who understood the context and the process and to be able to bounce ideas off of, or to acknowledge that it's OK to feel, like, upset about whatever you heard in an interview or something like that. (RA11)

2.5 Seek input from RAs throughout the study. In both of the focus group interviews, and in six of the individual interviews, the RAs identified themselves as an untapped resource. They identified that RAs can be instrumental in a variety of tasks that are usually thought of as beyond the job description of a typical RA, such as identifying important or unique results that may not be captured in the data analysis process. The RAs also felt that they could be an important source of information about study protocols and revisions, based on their experiences of data collection. In addition, RAs could be an important source of information about what participants are not being asked.

It's been really interesting to see sort of the gaps maybe the questions could have included. And just in terms of ... the design of the online tools, what sorts of things might have been done to better capture the data that people were reporting. (RA2)

2.6 Ensure that a smooth transition occurs between RAs. We did have some RAs who left the study, and others were hired after the project began. The RAs, especially those hired later in the study, identified the importance of investigators ensuring that RAs keep good records of their completed and incomplete activities. In addition, RAs discussed the importance of passing along information to newly hired RAs and spending time training them. Ideally, there would be an overlap between a RA who leaves and a new RA. "That's been challenging, trying to get information from a person who has left, and trying to find all the information" (RA10).

2.7 Educational credentials are not the only important factor in determining who will be an effective RA. Our RAs exhibited diversity in age, educational background, and applied experiences. A small number of RAs discussed the issue of differences between RAs, and suggested that this should influence how RAs are treated. "Someone who works in the health industry for 29 years has a very different take on a situation than someone who has just finished their undergrad" (Focus Group 1). In one focus group interview and two individual interviews, RAs identified that researchers should not focus solely on educational qualifications when hiring RAs, but that the unique contributions of each RA should be recognized. The ability of the RA to perform complicated tasks, such as interviewing participants with chronic health conditions, requires skills that do not necessary develop through formal educational experiences. Personal experiences, such as 
living with a person with a chronic health condition, and the personality of the RA, can also be important attributes. "You are dealing with people who are ill, who are struggling, and so when you go to hire RAs, I think that that's something you have to look for, the ability to be personable and connect" (RA13).

2.8 Pay for all hours worked and expenses incurred. In some cases, RAs identified some expenses related to the research project for which they did not receive reimbursement, including Internet time when working from home, purchasing batteries, and purchasing USB drives. In addition, some RAs identified that they worked unpaid over-time hours. In the latter cases, the RAs felt positive about contributing additional time for the good of the project as a whole. "I honestly put in more hours in some places than I should, but I just ended up doing it because I thought, well, it's not just about the money" (RA13).

\section{Discussion}

Much of the content of our results relates to the dual roles of the RA as both trainee and employee. Initial training and ongoing support is necessary in order to fulfill both of these roles. While the need for initial training for RAs is emphasized by other researchers (Naufel \& Beike, 2013; Rossouw \& Niemczyk, 2013), we used an extensive training process. Our emphasis on a relatively formalized training process at the beginning of employment of RAs was motivated by having a large number of RAs involved with our project at multiple sites. Standardization of training ensured consistency with data gathering procedures.

One of the most important themes we identified on either training or ongoing support for RAs relates to the need to provide guidance about the interactions between RAs and study participants. Other researchers have also identified the need for faculty mentors to provide specific guidance on the roles and responsibilities of RAs (Cambron \& Evans, 2003; Rossouw \& Niemczyk, 2013), but our results are particular to the relationship with study participants. The prominence of text on the relationship between RAs and participants may have emerged because we examined the everyday experience of living with and managing a chronic health condition. Thus, the questions were often quite personal and evoked various emotions from both study participants and RAs. Naufel and Beike (2013) identified several risks that RAs can experience, including psychological risk, which can result in emotional distress due to either the content of the research or the process of collecting data; this is especially evident for RAs who have repeated exposure to distressing situations. Many of our RAs engaged in multiple telephone or in-person interviews with the same participant over time, and they often became very aware of the participant's personality and circumstances. In addition, the RAs employed in our study either had an extensive background in working in health care and/or human services or were training to work in these fields, and were sensitized to the importance of relationships between professionals and clients. Thus, it is not surprising that issues about relationships between RAs and study participants emerged so strongly. RAs need to have the option of not participating in research activities they find distressing, or of reducing the amount of time spent conducting distressing duties (Naufel \& Beike, 2013).

Our findings further highlight the importance of what Earley (2007) terms developing a student's identity as a researcher, or how a RA's identity may influence her or his work as a researcher. Involvement in a research project can help RAs understand themselves 
more deeply and influence their work as professionals. Our results indicate that researchers exploring personal and sensitive topics should devote considerable time and attention to the task of helping RAs to manage their own responses and reactions to people who often face multiple life challenges. It is clear that in studies like ours, faculty mentors need to prepare RAs to be able to handle and react appropriately to unique and often unpredictable situations that occur during the research process, especially in situations of data collection with people experiencing serious health challenges (Earley, 2007; WulfAnderson et al., 2013). How to support the development of these abilities in RAs is much less clear and is worthy of further investigation.

It is clear that training RAs to complete their specific assigned tasks is crucial, but our RAs desired a much broader understanding of the study. Wulf-Andersen and colleagues (2013) identified that student researchers wish to feel let into the research process, such as by being welcomed and acknowledged, and feeling confident about being there. Our results extend these concepts further, to clarifying issues of accountability for study participants and sharing dissemination plans. Our RAs felt very accountable to the participants, and they wanted a clear understanding that the results of the study would make a difference. We recommend that researchers communicate to RAs a clear plan of dissemination of research results to various audiences, including the participants themselves and other researchers. In addition, researchers need to communicate to RAs the potential for the study to influence change in the actions of policy makers, policy administrators, and service providers (Bogenschneider, 2014).

As with other paid positions, RAs should be provided with regular feedback on the performance of their assigned duties (Naufel \& Beike, 2013). The RAs in our study offered interesting insights into various methods of delivering feedback to faculty mentors, and they even identified mechanisms for receiving feedback, including methods for RAs to anonymously request feedback. Some RAs in our study highly valued the opportunity to request feedback, while others preferred to have investigators initiate the feedback. Some RAs appeared quite comfortable working in a hierarchical system, while others enjoyed the more collegial and flatter hierarchy utilized in this project. Incorporating opportunities for feedback to any RAs working off-site or outside of regular office hours emerged as salient findings. Not all RAs desired the same amount or type of feedback mechanisms. Whiteside and colleagues (2007) recommend that in addition to having weekly team meetings, it is useful to hold individual meetings with each RA in order to elicit feedback and to allow the RA the opportunity to identify issues with the research project. It is clear that faculty mentors should implement a variety of ways to provide feedback to RAs, and feedback should be as individualized as possible, based on the preferences and needs of each RA. However, the challenge lies in faculty members' ability to balance time spent mentoring RAs in learning the research process versus ensuring that the research goals are achieved (Rossouw \& Niemczyk, 2013). This can be facilitated by the involvement of multiple faculty members to provide support to RAs, as in our study. Edwards (2009) identified that RAs benefit from the involvement of more than one faculty supervisor, and RAs ideally learn and work in an environment that is a research community.

Our research indicates that in addition to RAs having effective communication with the research team as a whole, investigators should provide opportunities for RAs to communicate directly with each other. The process of conducting research can be a solitary 
and isolating experience (Earley, 2007), and both peer and faculty member support can be useful to RAs (Grundy, 2004; Wulf-Andersen et al., 2013). Our RAs immensely appreciated the support they received from other RAs, perhaps due to being spread across three study sites and not always having the opportunity for a great deal of face-to-face interaction. However, these interactions can be facilitated using various electronic tools in addition to in-person communication.

Our RAs belonged to two groups based on their demographic characteristics, and this may not be typical of RAs involved in other studies, especially having a group of RAs over the age of 35 with significant professional experience in health and human services. Many of our RAs had worked as professionals before pursuing a graduate degree. Little research focuses on the issue of the age, prior training, and accumulated professional experience of RAs. However, Rossouw and Niemczyk (2013) recommend that less-experienced RAs not be relegated to basic administrative tasks. In projects like ours, which relied on a great deal of technology in data collection, this may be a particular aspect of research in which younger RAs may exhibit particular skills. Many of our younger RAs belong to a generation of people who use information and communication technology differently than older people, such as how they interact with others, and their proficiency with multitasking (Kubiatko, 2013). However, faculty mentors should not expect that RAs will know how to use specific technology based on age alone, especially when it comes to using specialized hardware or software for data collection, or knowing what to do if they encounter problems with the technology. It is clear that initial and ongoing technical support needs to be provided to RAs of all ages.

Our findings may not be applicable to RAs involved in other types of data collection with different study populations. Our RAs had direct contact with study participants through face-to-face and/or telephone interviews. In addition, we collected data over several months with people who have chronic health conditions. Thus, the experiences of our RAs may be quite different from those of RAs without this same direct contact with study participants or who collect data from people who do not have significant health issues.

It is very obvious that RAs are an integral part of many different types of research, including having key roles in data collection and knowledge production (Hobson et al., 2005). It is important for researchers to be aware that students employed as RAs are motivated not only by monetary rewards but also by the knowledge and experience that they gain (Niemczyk, 2010; Rossouw \& Niemczyk, 2013). Faculty members need to be diligent in ensuring that payment accounts for total hours worked, as RAs may be willing to invest additional time and may be reluctant to report this time because of the value of the work to trainees (Rossouw \& Niemczyk, 2013). Researchers need to be cognizant that the trainee aspect of being a RA is as important as the employee aspect. Thus, the paid RA role may be more similar to other contexts in which faculty mentoring occurs, such as supervising a graduate student's thesis or collaborating on a publication (Humble et al., 2006; Paglis, Green, \& Bauer, 2006).

Researchers may be surprised at the amount of information that RAs learn, not only about the research process and the development of research skills, but also about the topic of study, and how they integrate new knowledge with their prior learning and their plans for future employment. This is similar to Earley's (2007) concept of identity development through the process of conducting research. Thus, faculty mentors need to ensure that RAs' trainee role is not overshadowed by the employee role. 
Our results contribute significantly to the understudied topic of RAs hired to work on paid, faculty-driven, funded research projects (Rossouw \& Niemczyk, 2013). Our findings provide valuable insights for new and experienced faculty members who employ RAs in their research, and for others who supervise RAs, such as research managers or research co-ordinators, to ensure that appropriate training and ongoing support is provided to meet the needs of RAs with diverse backgrounds. Our results may contribute to the development of more formal regulations to ensure that RAs have greater protection as both trainees and employees (Rossouw \& Niemczyk, 2013). Our results are also relevant for RAs themselves to prepare for the role of being a RA, and especially for those working on studies involving collecting data from people dealing with significant health challenges.

\section{References}

Bogenschneider, K. (2014). Family policy matters: How policymaking affectsfamilies and what professionals can do (3rd ed.). New York, NY: Routledge.

Braun, V., \& Clarke, V. (2006). Using thematic analysis in psychology. Qualitative Research in Psychology, 3(2), 77-101. doi:10.1191/1478088706qpo63oa

Cambron, J. A., \& Evans, R. (2003). Research assistants' perspective of clinical trials: Results of a focus group. Journal of Manipulative and Physiological Therapeutics, 26(5), 287-292. doi:10.1016/So161-4754(03)00044-7

Earley, M. A. (2007). Lessons learned from students' research experiences. Journal of Research Practice, 3(1), Article E1. Retrieved from http://jrp.icaap.org/index.php/jrp/ article/view/93/75

Edwards, B. J. (2009). It takes a village: Perceptions of the SFU Education research assistant experience (Unpublished doctoral dissertation). Simon Fraser University, Burnaby, BC.

Fereday, J., \& Muir-Cochrane, E. (2006). Demonstrating rigor using thematic analysis: A hybrid approach of inductive and deductive coding and theme development. International Journal of Qualitative Methods, 5(1), 1-11.

Grundy, A. L. (2004). Learning experiences and identity development as a research assistant (Unpublished master's thesis). Brock University, St. Catharines, ON.

Hey, V. (2001). The construction of academic time: Sub/contracting academic labour in research. Journal of Education Policy, 16(1), 67-84. doi:10.1080/02680930010009831

Hobson, J., Jones, G., \& Deane, E. (2005). The research assistant: Silenced partner in Australia's knowledge production. Journal of Higher Education Policy and Management, 27(3), 357-366. doi:10.1080/13600800500283890

Humble, Á. M., Solomon, C. R., Allen, K. R., Blaisure, K. R., \& Johnson, M. P. (2006). Feminism and mentoring of graduate students. Family Relations, 55(1), 2-15. doi:10.1111/ j.1741-3729.2006.00352.x

Kang, D. H., Davis, L., Habermann, B., Rice, M., \& Broome, M. (2005). Hiring the right people and management of research staff. Western Journal of Nursing Research, 27, 1059-1066. 
Krueger, R. A., \& Casey, M. A. (2000). Focus groups: A practical guide for applied research. Thousand Oaks, CA: Sage.

Kubiatko, M. (2013). The comparison of different age groups on the attitudes toward and the use of ICT. Educational Sciences: Theory \& Practice, 13(2), 1263-1272.

Luborsky, M. R. (1994). The identification and analysis of themes and patterns. In J. F. Gubrium \& A. Sankar (Eds.), Qualitative methods in aging research (pp. 189-210). Thousand Oaks, CA: Sage.

Madden, A. D. (2009). Managing for the ideal research environment. Journal of Higher Education Policy \& Management, 31(3), 271-282. doi:10.1080/13600800902974369

McGinn, M. K., \& Niemczyk, E. K. (2013). Research practice in research assistantships: Introducing the special issue on research assistantships. Journal of Research Practice, 9(2), Article E2. Retrieved from http://jrp.icaap.org/index.php/jrp/article/view/384/310

McGinn, M. K., Niemczyk, E. K., \&Saudelli, M. G. (2013). Fulfilling an ethical obligation: An educative research assistantship. Alberta Journal of Educational Research, 59(1), 72-91.

Miller, M., Stephens, J. B. (1998). A partnership paradigm: A case study in research assistant and faculty interaction. Journal of Staff, Program and Organization, 15(3), 111-119.

Moore, J., Scarduzio, J. A., Plump, B., \& Geist-Martin, P. (2013). The light and shadow of feminist research mentorship: A collaborative autoethnography of faculty-student research. Journal of Research Practice, 9(2), Article M8. Retrieved from http://jrp.icaap. org/index.php/jrp/article/view/343/309

Naufel, K. Z., \& Beike, D. R. (2013). The ethical treatment of research assistants: Are we forsaking safety for science? Journal of Research Practice, 9(2), Article M11. Retrieved from http://jrp.icaap.org/index.php/jrp/article/view/36o/318

Niemczyk, E. K. (2010). Expanding the research horizon in higher education: Master's students' perceptions of research assistantships (Unpublished master's thesis). Brock University, St. Catharines, ON.

Nishimura, C., Takahashi, R., Miyamoto, S., Saito T., Kanemaru, A., \& Liehr, P. R. (2003). Lessons learned as a research assistant studying ambulatory blood pressure in elderly Japanese stroke patients. Nursing and Health Sciences, 5, 51-57. doi:10.1046/ j.1442-2018.2003.00134.x

Paglis, L., Green, S., \& Bauer, T. (2006). Does adviser mentoring add value? A longitudinal study of mentoring and doctoral student outcomes. Research in Higher Education, 47(4), 451-476. doi:10.1007/s11162-005-9003-2

Rossouw, J. P., \& Niemczyk, E. K. (2013). A dual perspective on risks and security within research assistantships. Journal of Research Practice, 9(2), Article M10. Retrieved from http://jrp.icaap.org/index.php/jrp/article/view/358/317

Versnel, J., Packer, T., Weeks, L. E., Brown, J., Godwin, M., Hutchinson, S., . . . Warner, G. (2013). The everyday experience of living with and managing a neurological condition (the LINC study): Study design. BMC Neurology, 13(30), 1-11. Retrieved from www.biomedcentral.com/1471-2377/13/30 
Whiteside, U., Pantelone, D. W., Hunter-Reel, D., Eland, J., Kleiber, B., \& Larimer, M. (2007). Initial suggestions for supervising and mentoring undergraduate research assistants at large research universities. International Journal of Teaching \& Learning in Higher Education, 19(3), 325-330.

Wulf-Andersen, T., Holger Mogensen, K., \& Hjort-Madsen, P. (2013). Researching with undergraduate students: Exploring the learning potentials of undergraduate students and researchers collaborating in knowledge production. Journal of Research Practice, 9(2), Article M9. Retrieved from http://jrp.icaap.org/index.php/jrp/article/view/351/316

\section{Contact information}

Lori E. Weeks

School of Nursing

Dalhousie University

lori.weeks@dal.ca

Lori Weeks is an associate professor in the School of Nursing at Dalhousie University. Her training is in gerontology and family studies, and her primary research interests are directed at care and support services for older adults and their caregivers, and factors affecting the health of seniors. Her research often focuses on vulnerable populations. Dr. Weeks also focuses on the scholarship of pedagogy, including improving the experiences of students completing field placements and distance courses.

Michelle Villeneuve is a senior lecturer in occupational therapy at the University of Sydney, Australia, and holds a cross-appointment with the School of Rehabilitation at Queen's University. Michelle is an experienced academic instructor with extensive background in international development, curriculum design, and evaluation. Michelle's research uses participatory and action-oriented approaches for improving multi-agency and interprofessional collaborative work to support the participation outcomes of people with disabilities and their families.

Susan Hutchinson is an associate professor of leisure studies in the School of Health and Human Performance at Dalhousie University. Her research focuses on the ways in which meaningful leisure engagement can be a resource in "living well" with a chronic mental or physical health condition or acquired disability. Within this area, her interest is in leisurebased coping and self-management.

Kerstin Roger is an associate professor in Community Health Sciences at the University of Manitoba. She holds a Master's in Applied Psychology and a PhD in Sociology and Equity Studies in Education. Her current research focuses on the family and community interfacing with the health care system, social services and aging, as well as social aspects of living with a neurological condition across the lifespan. She is the founder and Director of the Qualitative Research Group, a community of practice with over 200 members across Canada and internationally. 
Joan Versnel is an associate professor in the School of Occupational Therapy at Dalhousie University. Her PhD is in Cognitive Studies/Educational Psychology from Queen's University. Her research has explored the theme of shared decision-making and negotiated goal setting with youth in educational and health care settings. Early preparation for chronic disease self-management has been an element in several of her research initiatives, including self-advocacy for adolescents, negotiation of accommodations in the workplace for individuals with chronic illness and disability, as well as enhancing interprofessional collaboration in pre-licensure health professional education programs.

Tanya Packer is Professor and Director in the School of Occupational Therapy at Dalhousie University. As an occupational therapist, her interest and expertise have always been focused on the everyday experience of living with and managing life with a disability or long-term condition. Her international career has seen her teaching and conducting research in many continents: developing the first occupational therapy education program in Russia, undertaking community-based rehabilitation in China and India, studying accessible tourism in Hong Kong, and conducting research into the ways people manage chronic and long-term conditions in Western Australia and Canada. 